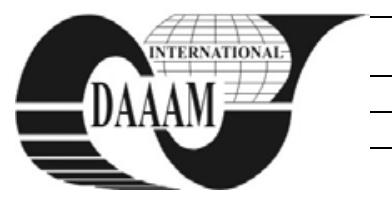

\title{
ALGORITHM FOR SIMULATION OF THE COUPLED ELECTROMAGNETIC AND THERMAL PHENOMENA
}

\author{
NAGY, S[tefan]; LEUCA, T[eodor]; MICH - VANCEA, C[laudiu] \& NAGY, A[drian]
}

\begin{abstract}
The paper presents the computation algorithm and a software package (NAG) for the eddy currents and Joule losses problem in a probe using the integral equation method for the current density and the finite method for the thermal field diffusion problem. The software package is based on a mesh generation procedure. The meshing algorithm allows the use of a single mesh for solving both the eddy currents problem and the thermal problem using the finite differential method. The software allows, beside the structure and rotation symmetry, the interactive development of a mesh that could have any shape.
\end{abstract}

Key words: numerical analysis, integral equation method, finite differential method

\section{INTRODUCTION}

The computation of losses through eddy currents is a problem of major interest in the field. For most cases, these losses are not wanted and specialists try to minimize their effect as much as possible. In other cases, they are useful due to their caloric effect, allowing the controlled and local heating of conducting materials. In order to determine the losses due to the eddy currents it is necessary to compute the electromagnetic field in the structures with conductor properties, thus solving complicated quasi-stationary regime problems.

The computation of the transient thermal regimes in materials with temperature dependant properties can raise numerical problems, especially for phase transformations, like melting or solidification. The traditional methods, based on the local form of the thermal conduction equation, using the temperature dependant specific heat to represent the thermophysical properties, become inadequate for phase transitions. Fluerasu 2008, present a different approach, based on using the integral form of the thermal conduction equation and the material's caloric state equation. This method ensures the convergence of the iterations involved by the non-linearities.

The thermal diffusion equation is nonlinear, due to the presence of the solidification layer. In order to illustrate the suggested procedure we developed a $2 \mathrm{D}$ program, but the method can also be extended to 3D structures and non-linear structures with ferromagnetic materials (Ciric, 2009).

Aiello 2008, propose a hybrid method, called finite element method-Dirichlet boundary condition iteration (FEM-DBCI), for the computation of time-harmonic eddy current problems inside a conductor heated by coils in 3D open-boundary geometry. The method couples a differential equation for the interior problem in terms of the electric field with an integral equation for the exterior one. The global algebraic system is efficiently solved in an iterative way.

In this paper we try o develop a computation procedure to solve the coupled electromagnetic and thermal problem (using two different methods) through a software package. Both methods use the same mesh, the Joule losses calculated in the electromagnetic problem will be used as heat sources for the thermal problem.

Formulating the electromagnetic field problem in the form of the integral equation of the eddy currents has the following advantages:
- Only the conducting domains are divided in sub-domains and the number of unknowns associated to them are relatively small;

- Moving parts can easily be taken into account. If the excitation coils are moving, with imposed current densities, then only the free term of the integral equation is changing. If the part with induced currents is moving, then only a part of the system coefficients (associated to part coupling) are changing in time. The speed is not involved, the reference systems are the Lagrange ones, coupled to the moving parts;

- There is no need to define the boundaries for the computation domain, like for the differential methods.

Numerical simulation use a complex mathematic model that treats separately the electromagnetic field phenomena coupled with the thermal field phenomena. This mathematical model was described by the author in (Nagy, 2008).

\section{STRUCTURE OF THE NAG SOFTWARE PACKAGE AND RESULTS}

In order to determine the eddy currents and the Joule losses in the probe we used the integral equation method of the current density, developing a software package called NAG.

The algorithm was implemented in Fortran, that is a powerful programming language necessary for elaborate computations for numerical modeling of coupled electromagnetic and thermal phenomena taking place in the casting processes at the transition from liquid phase to solid phase $(* * * 2008)$.

The flow-chart of the software program that presents the computation algorithm for the coupled electromagnetic and thermal diffusion fields is listed in fig. 1.

The software package developed to solve the electromagnetic problem has the following components:

- NAGDATERM - input of the geometrical data, resistivity of the molten, crucible and bottom; input of the number of sections for the automatic generation of the volume elements. The program allows the interactive generation of any mesh shape.

- NAGIND - setup of the coil abscissa and the step counter, input of the movement distance of the coil and the step along the Oy axis.

- GENVOL - drawing of the mesh.

- NAGPREG (or NAGPREG5) - processing of the input data: computing the weight centers of the sub-domains and the current densities for the boulders.

- NAGAJ (or NAGAJ5) - computing the vector potentials produced by the eddy currents in the weight centers of the subdomains (the $\mathrm{N} \times \mathrm{N}$ weight coefficients matrix).

- NAGMATR (or NAGMATR5) - computes the system matrix and inverts it.

- NAGA0 - determines the free terms.

- NAGSLVD - computes the eddy currents in the subdomains (the module, real and imaginary components) and the loss density in the sub-domains.

- NAGCORD - modifies the position of the coil (and the initial data through NAGIND) and draws the field lines, fig. 2. 


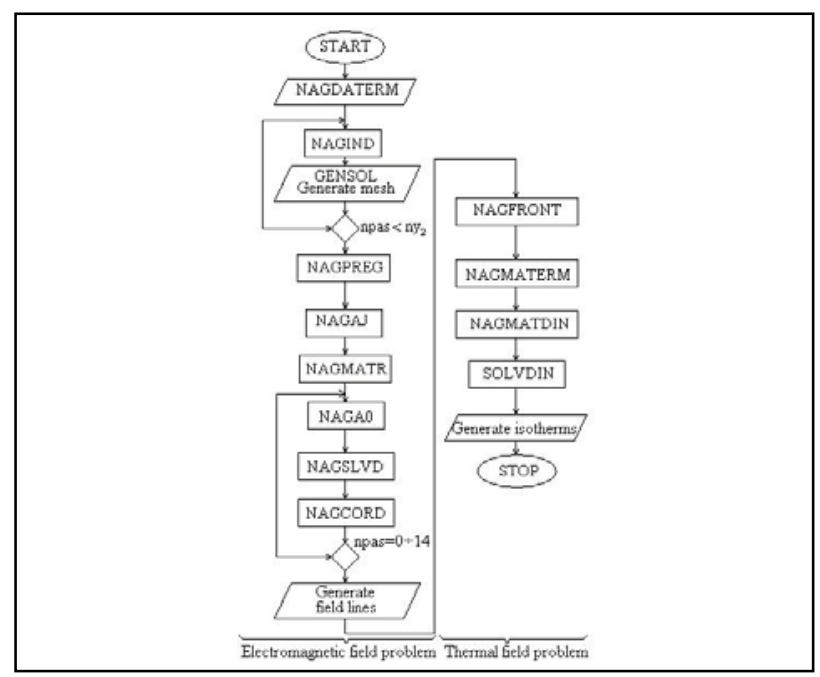

Fig. 1. Flow-chart of the software package

The first six programs are required to run only once, while the last three are repeated for each time step.

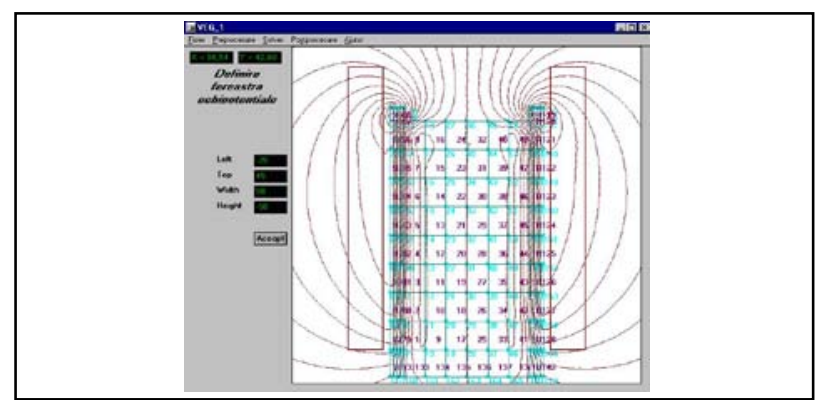

Fig. 2. Electromagnetic field lines

The eddy currents vary in time. In each point they have a sinusoidal variation, but not on phase. Because the current varies, we get a time variation of the field lines for each position (step) of the probe. The probe has 14 positions (npas $=0 \div 14$; npas $<$ ny2, where y2 is the number of sections on the $y$ axis for the probe). The angle on the current sinusoid can take values from $0 \div 5$.

The thermal field diffusion problem is a very complicated one. From the modern thermal computation methods we chose the finite differential method, because of the following advantages:

- We can use the same mesh from the electromagnetic problem to solve the thermal field, the computation domain being the conducting area;

- Can be easily applied for thermally non-homogenous environments (probe, molten). Can take into account the strong non-linearity due to the thermal dependence of the thermal capacity in the phase variation area. In this case we cannot apply the boundary elements.

The time-framing is done using the Crank-Nicholson algorithm, increased attention being turn to the term that contains the thermal capacity. It is adjusted iteratively depending on the temperature. The time step is chosen so that the procedure is stable. The boundary conditions are varying in time according to the movement of the probe, because beneath the inductor there is the cooling system.

The software package developed to solve the thermal problem contains:

- NAGFRONT - sets the boundary points, the boundary types and boundary conditions (fixed or varying);

- NAGMATERM - build the part of the coefficient system and free term matrix that does not vary in time;

- NAGMATDIN - build the part of the coefficient system and free term matrix that varies in time;

- SOLVDIN - solves the system iteratively and draws the isotherms, fig 3.

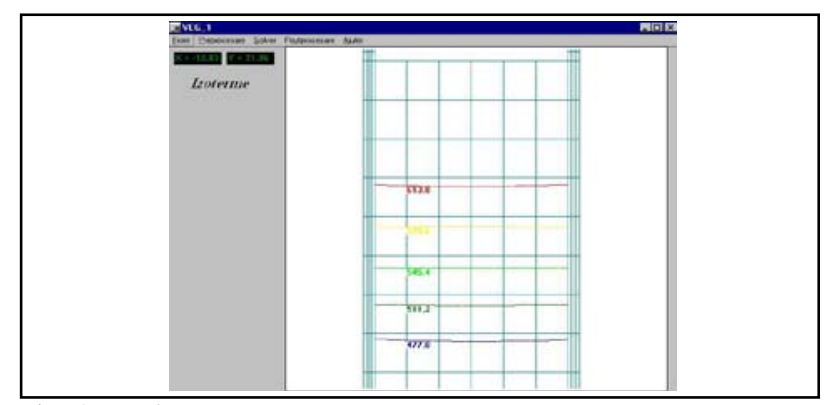

Fig. 3. Isotherms

\section{CONCLUSION}

Studying the evolution of the coupled electromagnetic and thermal phenomena in a probe, like the phase variation layer evolution during the controlled solidification process, allowed us to develop the following:

- a method to compute the eddy currents and the Joule losses in the probe. The method takes into account the movement of the probe inside the inductor. It enables us to solve the eddy currents' integral equation.

- a method to determine the thermal field during the thermal diffusion regime. The problem raises two major difficulties: the non-linearity due to the temperature dependence of the thermal capacity and time varying boundary conditions. We used the finite differential method, thus the mesh used for the eddy currents problem can also be used for solving the thermal problem. The time-framing is done using a Crank-Nicholson procedure. The non-linear algebraic system is checked iteratively, adjusting the values for the thermal capacity.

- a software package (approximately 6000 code lines in FORTRAN) that solves both electromagnetic and thermal problems. Thus, we obtain information about the current density and the layout of the Joule losses for each step of the probe inside the inductor, the field lines for the magnetic induction, the thermal field at any time during the controlled solidification process and the isotherms' evolution.

The presented algorithm helps to develop a procedure for determining the phase variation surface in the controlled solidification process that will allow the achievement of further improvement and optimization in designing the casting installations that will provide products with a homogeneous crystalline structure.

\section{REFERENCES}

Aiello, G.; Alfonzetti, S., Dilettoso, E., Salerno N. (2008) Thermal analysis of an eddy-current heated piece by means of the FEM-DBCI method, COMPEL: The Int. Journal for Comp. and Mathematics in Electrical and Electronic Engineering, Vol. 27, No. 2, pp. 427-435, ISSN 0332-1649

Ciric, I.; I.F. Hantila, I.F., Maricaru, M., Marinescu, S. (2009). Efficient analysis of the solidification of moving ferromagnetic bodies with eddy-current control, IEEE Trans. on Mag., Vol. 45, No. 3, pp. 1238-1241, ISSN: 0018-9464

Fluerasu, C.; Fluerasu, Corina (2008). Calcul des regimes transitoires thermiques dans des materiaux avec proprietes dependantes de la temperature, Revue Roumaine des Sciences Techniques Serie Electrotechnique et Energetique, Vol. 53, No. 3, pp. 269-278, ISSN: 0035-4066

Nagy, St.; Leuca, T., Mich-Vancea, C. (2008). Numerical modeling of the coupled electromagnetic and thermal fields in the controlled solidification processes, ICEM'08- XVIII Int. Conference on Electrical Machines, 6 - 9 Sept., Vilamoura, Portugal, ISBN: 978-1-4244-1736-0

*** (2008) http://www.cs.mtu.edu/ shene/COURSES/ cs201/NOTES/intro.html - Basic Fortran, Accessed on: 2008 